\title{
The post-lunch effect of regular and decaffeinated coffee on psychomotor performance: a randomized double-blind cross-over study
}

\author{
Suganthi S. Ramachandran ${ }^{1 *}$, Vijay L. Kumar ${ }^{1}$, S. N. Dwivedi ${ }^{2}$, \\ Vishwajeet Singh ${ }^{2}$, Pooja Gupta ${ }^{1}$
}

${ }^{1}$ Department of Pharmacology, ${ }^{2}$ Department of Biostatistics, All India Institute of Medical Sciences, Ansari Nagar, New Delhi, India

Received: 03 June 2021

Accepted: 07 July 2021

*Correspondence:

Dr. Suganthi S. Ramachandran,

Email: drsugiram@yahoo.in

Copyright: (c) the author(s), publisher and licensee Medip Academy. This is an open-access article distributed under the terms of the Creative Commons Attribution Non-Commercial License, which permits unrestricted non-commercial use, distribution, and reproduction in any medium, provided the original work is properly cited.

\begin{abstract}
Background: This study aimed to compare the effect of regular coffee and decaffeinated coffee on psychomotor performance in healthy volunteers during post-lunch period.

Methods: In this randomized double-blind cross-over study, adult healthy volunteers were given hot coffee $(3 \mathrm{~g}$ each of regular or decaffeinated coffee) during post-lunch period. Psychomotor functions (critical flicker-fusion frequency (CFF), choice reaction time (CRT) and error count in hand-steadiness test (HST)), blood pressure and heart rate were measured pre-lunch, pre-coffee (1-hour post-lunch) and 1-hour post-coffee consumption. Subjective ratings of sleepiness and mood were also assessed during post-lunch sessions.

Results: The mean age of the participants $(n=16)$ was $27.4 \pm 2.7$ years with a male: female ratio of 7:9. There was no significant deterioration in psychomotor performance post-lunch when compared to pre-lunch on both the days. The mean CFF, CRT, errors committed in HST and cardiovascular parameters did not differ significantly between regular coffee and decaffeinated groups during post-lunch sessions. There was no significant difference in values of cardiovascular parameters as well as subjective ratings of sleep and mood between two groups.

Conclusions: In healthy adult individuals' consumption of both regular coffee and decaffeinated coffee during the postlunch period did not affect psychomotor performance.
\end{abstract}

Keywords: Caffeine, Coffee, Choice reaction time, Critical flicker fusion frequency, Hand steadiness test, Psychomotor performance

\section{INTRODUCTION}

Post-lunch dip in psychomotor performance is a commonly observed phenomena that has been linked to a 12-hour harmonic in 24-hour circadian rhythm. ${ }^{1,2}$ It is a common practice to consume coffee or tea during this period in order to enhance the work performance. Caffeine present in coffee or tea is well known to positively influence various domains of psychomotor performance like reaction time, concentration, attention time and vigilance. ${ }^{3-5}$ Besides, acute and chronic effects of caffeine have also been reported on higher order central nervous system functions like cognition and mood. ${ }^{4-6}$ An improvement in learning, decision-making and real-world activities such as automobile and aircraft operation in sleep-deprived individuals has also been reported following caffeine intake. ${ }^{7}$ Bioactive components of coffee other than caffeine, its sensory properties like aroma, taste, visual appearance, anticipation and conditioning effect associated with coffee consumption have also been suggested to contribute to this beneficial effect when evaluated at various time points of the day. ${ }^{8-11}$

Addition of caffeine tablets to decaffeinated coffee has also been found to improve attention span and mood of the 
individual during post-lunch period. ${ }^{2}$ Coffee is either consumed as black coffee in plain water or with milk and sugar. Milk and sugar reduce the bitterness of coffee without affecting the bioavailability of phenolic acids present in it and is the traditional way of coffee consumption in India. ${ }^{12}$ It was hypothesized that regular coffee in comparison to decaffeinated coffee (both with milk and sugar) would improve psychomotor performance in adult healthy volunteers during post-lunch period. Hence, the primary objective of this study was to compare the effect of regular and decaffeinated coffee on psychomotor performance during post-lunch period. Secondary objectives were to compare the effect of lunch on psychomotor performance and to compare the effect of regular versus decaffeinated coffee on cardiovascular, sleep and mood parameters during post-lunch period.

\section{METHODS}

A randomized cross-over study was conducted in the Clinical Pharmacology Research laboratory, at Department of Pharmacology after approval of institute ethics committee. The study was conducted according to Declaration of Helsinki and Good Clinical Practice guidelines. It was registered with Clinical Trials Registry of India (CTRI/2019/02/017763).

\section{Study population}

The study included 16 post-graduate students, of either gender, in the age range of 21-35 years, who provided written informed consent. Their demographic and clinical characteristics are given in Table 1 . The sample size was calculated in order to have $80 \%$ chance of detecting a decrease in reaction time of 40 milliseconds in regular coffee group versus decaffeinated coffee group with a standard deviation of 40 milliseconds and significance at the $5 \%$ level. $^{2}$ It excluded individuals who were current smokers (smoked at least 100 cigarettes in the past and also currently smoke cigarettes); with medication intake (especially drugs causing psychomotor impairment like anti-histamines, benzodiazepines, caffeine containing medicinal preparations, CYP1A2 inducers and inhibitors); with cough and cold for past 1 week; with caffeine use disorder as per Diagnostic and statistical Manual 5 (DSM 5) criteria; with any other concurrent illness like hypertension and diabetes mellitus; consuming more than 4 cups of any type of coffee every day; who have never consumed caffeinated beverages; who were pregnant or lactating. ${ }^{13,14}$

\section{Randomisation and allocation concealment}

A computer-generated permuted block randomization was performed by statistician using STATA version 14.2 software. A third person who was not involved in the study had placed the coffee pouches inside the sequentially numbered sealed opaque envelopes as per randomization sequence generated. The investigator and participants were blinded to the study intervention.
Table 1: Demographic and clinical characteristics of participants.

\begin{tabular}{|c|c|}
\hline Characteristics & $\begin{array}{l}\text { Participants } \\
(\mathrm{n}=16)\end{array}$ \\
\hline Age in years (mean \pm SD) & $27.4 \pm 2.7$ \\
\hline Sex (male: female) & $7: 9$ \\
\hline BMI in kg/m² $($ mean \pm SD) & $24.4 \pm 3.3$ \\
\hline History of Myopia (n) (\%) & $10(62.5)$ \\
\hline $\mathrm{SBP}$ in $\mathrm{mm}$ of $\mathrm{Hg}($ mean $\pm \mathrm{SD})$ & $106.6 \pm 9.3$ \\
\hline DBP in $\mathrm{mm}$ of $\mathrm{Hg}($ mean $\pm \mathrm{SD})$ & $69.5 \pm 7.0$ \\
\hline$H R$ in beats per minute $($ mean $\pm S D)$ & $80.3 \pm 7.7$ \\
\hline
\end{tabular}

\section{Study procedures}

All eligible participants gave written informed consent and were familiarized with the instruments used for psychomotor test while screening, one day prior to the study. Participants were advised not to consume any alcoholic beverages or caffeinated products from the previous night of the study day and not to do any strenuous physical activity on the study day. They were also advised to have a good night sleep. Out of sixteen participants, eight were randomly allocated to decaffeinated-regular coffee sequence and remaining were allocated to regulardecaffeinated coffee sequence with a minimum of threeday time interval (Figure 1). On the first day of study, participants reported to the clinical pharmacology laboratory around 10:00 am after having light breakfast around 8:00 am. Their history, physical examination, frequency of caffeinated product consumption as never, rare, seldom (once a week), occasional (few times a week), regular (on most days per week) and very regular (daily or multiple times daily) were captured in the study specific case record form. Majority of the participants (75\%) were regular or very regular tea or coffee drinkers. A pre-lunch assessment of psychomotor function and cardiovascular parameters was made between 10:00 and 11:00 am. The participants were provided standard vegetarian meal consisting of two pieces of whole wheat flour bread (roti) and one bowl of vegetables comprising of carbohydrate: $50 \%$, protein: $15 \%$ and fat: $35 \%$ with an approximate caloric value of $650 \mathrm{Kcal}$ at 1:00 pm and were encouraged to complete the lunch within half an hour. Pre-coffee assessments, coffee intake and post-coffee assessments were carried out at $2.30 \mathrm{pm}, 3.00 \mathrm{pm}$ and $4.00 \mathrm{pm}$ respectively as described in Figure 2. After intervention the participants were shown nature related videos for one hour. The cross-over part of the study was performed between day 3 and 8 with maximum participants $(75 \%)$ reporting on day 7 and followed the similar time schedule as in previous visit.

\section{Coffee preparation}

Three grams of granules of regular and decaffeinated coffee (single lot, Nescafe gold, Nestle products, India equivalent to $90-100 \mathrm{mg}$ and $3-12 \mathrm{mg}$ of caffeine 
respectively) were weighed and sealed in airtight ziplock bags. ${ }^{3,15}$ One packet of coffee, two sugar cubes ( $4.1 \mathrm{~g}$ each) and two milk powder sachets (1.8 g each; single lot, Nestle Everyday dairy creamer) were dissolved in a cup containing $150 \mathrm{ml}$ of hot water $\left(55\right.$ to $\left.60{ }^{\circ} \mathrm{C}\right)$.

\section{Psychomotor assessment}

The psychomotor assessment was carried out in a semi dark, quiet room. The order of administration of tests was Choice Reaction Time (CRT), Critical Flicker-fusion Frequency (CFF), and Hand-steadiness Test (HST) for all the sessions on both days and the time taken for completion of each test was 20 seconds, 8 minutes, and 2 minutes respectively.

\section{CRT}

It was measured as an indicator of sensori-motor processing of input-output response chain in central nervous system. ${ }^{10}$ The participant was administered a series of visual stimuli of red, green, blue and yellow LED lights at random using multiple CRT apparatus (INCO Ltd, Ambala, India). The time taken to switch off the light by pressing the button or by foot pedal was measured by a digital chronometer. The average of twenty readings was expressed as CRT in milliseconds.

\section{CFF}

It is an indicator of cortical arousal and alertness and denotes the ability of an individual to discriminate between flickering and steady state light beam. ${ }^{16}$ The frequency at which the individual starts perceiving the flickering light as fused light is called ascending threshold and vice-versa is known as descending threshold. CFF denotes the average of ascending and descending threshold and was measured by digital flicker fusion apparatus (INCO Ltd, Ambala, India). Three replicate measurements of ascending and descending thresholds for both red and green light source were recorded and the average of six recordings was taken as G-CFF for green light source and R-CFF for red light source and expressed in hertz $(\mathrm{Hz})$.

\section{HST}

It assesses the fine motor control where the participant inserts a probe in holes of decreasing radius $(9,7,6,5,3$ and $2 \mathrm{~mm}$ ) to a depth of $5 \mathrm{~mm}$ without touching the sides while holding for 20 seconds. The number of contacts of the probe with the side wall of hole was recorded by impulse counter in the digital hand steadiness tester (INCO Ltd, Ambala, India) and expressed as HST error count.

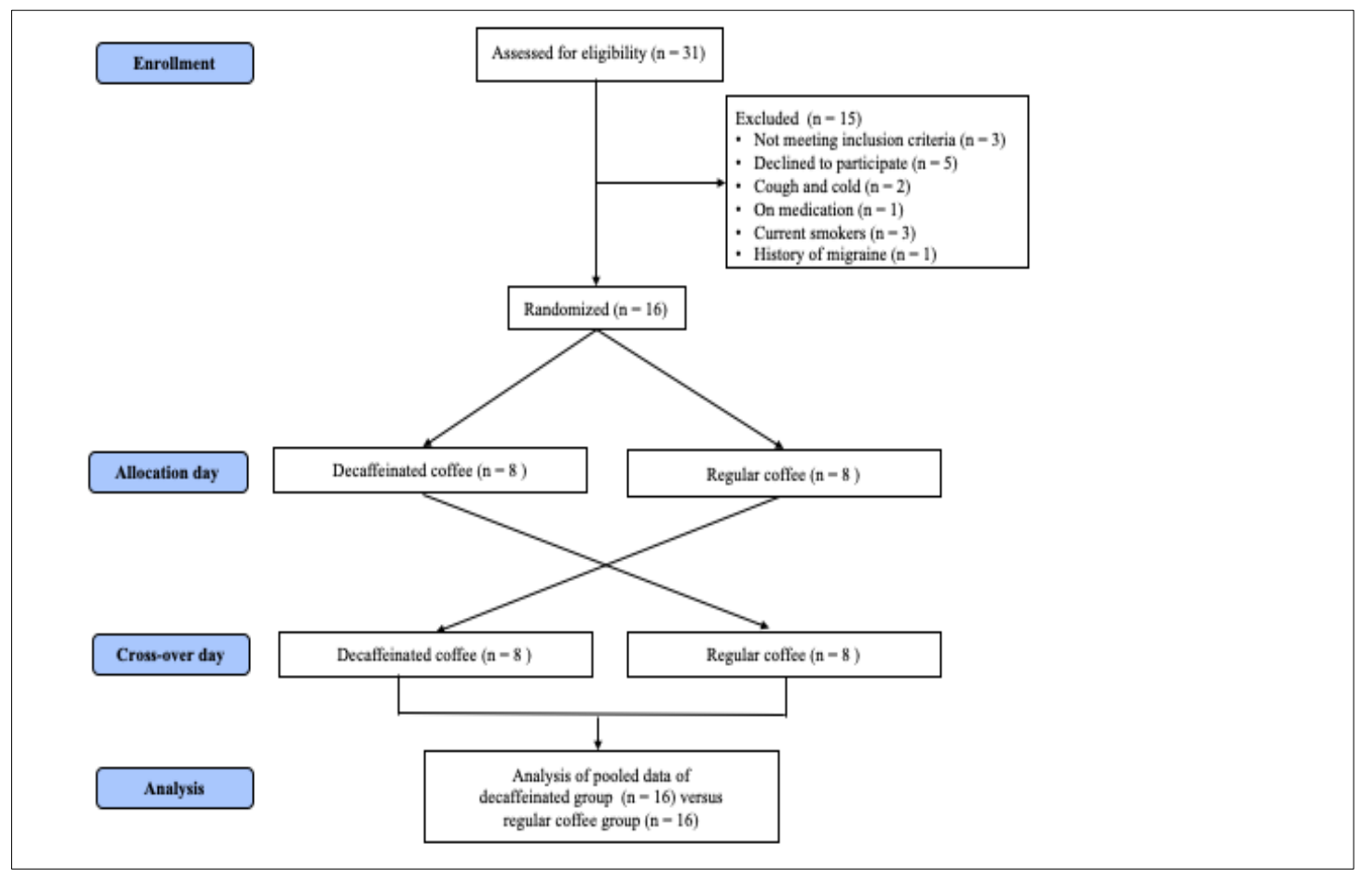

Figure 1: Participant enrolment and study design.

\section{Cardiovascular evaluation}

Systolic and diastolic blood pressures (SBP and DBP) and heart rate $(\mathrm{HR})$ were recorded in sitting position using Omron digital BP apparatus (Tokyo, Japan) and average of three readings was considered for analysis.

\section{Sleep duration and mood evaluation}

The participants were asked to rate their previous night sleep quality with a rating of 0 for bad quality to 10 for good quality. 
Subjective ratings of state of mind were recorded in a visual analogue scale of $0-10 \mathrm{~cm}$ during post-lunch period before and after coffee, viz. sleepiness (0) and awake state (10), anxious (0) and casual (10), happy (0) and sad (10), lethargic (0) and energetic (10).

\section{Statistical analysis}

As the study was a cross-over trial, the carryover effect (the residual effect caused by the previous treatment due to different order of administration of interventions) and period effect (the familiarization with study situation during cross-over day when compared to allocation day) had been initially assessed by unpaired t test and both were not found to be significant [carry over effect: $\mathrm{p}=0.518$, $0.055,0.166,0.324$ and period effect: $p=0.697,0.367$, $0.53,0.465$ for G-CFF, R-CFF, CRT and HST, respectively]. As the pre-coffee values between two groups were not found to be significant, the post-coffee values of psychomotor functions between regular and decaffeinated groups was analysed using paired t test (primary outcome measure). The same test was used in the analysis of pre-lunch and pre-coffee values as well as cardiovascular and subjective ratings of mood and sleep. Statistical tests were performed using STATA (version 14.2, StataCorp LP, College Station, TX: USA) and the data are expressed as mean \pm standard deviation (SD). The value of $p<0.05$ was considered statistically significant.

\section{RESULTS} Effect of decaffeinated and regular coffee on
psychomotor performance during post-lunch period

The psychomotor functions (primary outcome of the study) when evaluated in decaffeinated and regular coffee groups before and after coffee intake, did not reveal a significant change. The pre-coffee and post coffee values of G-CFF and R-CFF were found to be similar in both the groups $(p=0.318$ and $p=0.783$, respectively). A further evaluation of CRT in both the groups showed only a marginal decrease in post coffee values as compared to pre-coffee values though the difference was not found to be statistically significant $(\mathrm{p}=0.849)$. Similarly, in comparison to pre-coffee values, post coffee values of HST error count were also not significantly different in both the groups $(\mathrm{p}=0.92)($ Table 2).

\section{Effect of lunch on psychomotor performance}

The effect of lunch on psychomotor performance was evaluated both on allocation day and crossover day separately and the pre-lunch values were compared with post-lunch (i.e. pre-coffee) values. Table 3 shows that prelunch and pre-coffee values for G-CFF, R-CFF and HST error count were comparable on both allocation and crossover days. There was a slight change in pre-coffee values of CRT with respect to pre-lunch values on both the days though the difference was not statistically significant $(\mathrm{p}=0.666$ and $\mathrm{p}=0.559$, respectively).

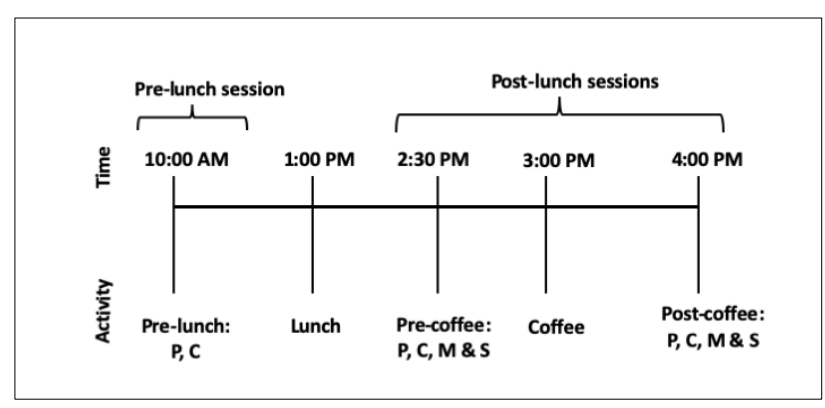

P: psychomotor function, (CFF: critical flicker-fusion frequency, CRT: choice reaction time, HST: hand steadiness test, EC: error count), C: cardiovascular parameters, (SBP: systolic blood pressure, DBP: diastolic blood pressure, HR: heart rate), M: mood rating, and $\mathrm{S}$ : sleep rating.

Figure 2: Time schedule for assessment of outcome parameters on a single allocation day.

\section{Effect of decaffeinated and regular coffee on cardiovascular parameters during post-lunch period}

The pre-coffee and post-coffee values of SBP, DBP and HR were found to be comparable in both decaffeinated and regular coffee groups and the mean difference of postcoffee values in SBP, DBP and HR between regular and decaffeinated coffee groups $(1.8 \mathrm{~mm}$ of $\mathrm{Hg}, 1.9 \mathrm{~mm}$ of $\mathrm{Hg}$ and -2.8 beats per minute) did not reach statistical significance $\quad(\mathrm{p}=0.364, \mathrm{p}=0.140$ and $\mathrm{p}=0.362$, respectively).

Table 2: Psychomotor performance in decaffeinated and regular coffee group during post-lunch period.

\begin{tabular}{|c|c|c|c|c|c|}
\hline \multirow{2}{*}{$\begin{array}{l}\text { Psychomotor test } \\
\text { parameters }\end{array}$} & \multicolumn{2}{|c|}{ Decaffeinated coffee $(n=16)$} & \multicolumn{2}{|c|}{ Regular coffee $(n=16)$} & \multirow{2}{*}{ P value } \\
\hline & Pre-coffee & Post-coffee & Pre-coffee & Post-coffee & \\
\hline G- CFF (Hz) & $40.8 \pm 2.7$ & $40.9 \pm 2.9$ & $41.0 \pm 3.4$ & $41.5 \pm 2.4$ & 0.318 \\
\hline R-CFF (Hz) & $40.2 \pm 2.8$ & $40.7 \pm 2.9$ & $40.4 \pm 2.4$ & $40.8 \pm 2.1$ & 0.783 \\
\hline CRT (milliseconds) & $521.6 \pm 100.5$ & $507.1 \pm 73.2$ & $519.9 \pm 67.9$ & $511.8 \pm 79.7$ & 0.849 \\
\hline HST error count & $81.9 \pm 24.0$ & $80.3 \pm 32.8$ & $74.8 \pm 29.5$ & $79.3 \pm 27.7$ & 0.92 \\
\hline
\end{tabular}

Values represent mean \pm SD. G-CFF: critical flicker-fusion frequency for green light source; R-CFF: critical flicker-fusion frequency for red light source; Hz: Hertz; CRT: choice reaction time; HST: hand steadiness test 
Table 3: Psychomotor performance during pre-lunch and pre-coffee period.

\begin{tabular}{|llll|}
\hline Psychomotor test parameters & Pre-lunch $(\mathbf{n = 1 6})$ & Pre-coffee $(\mathbf{n}=\mathbf{1 6})$ & P value \\
\hline Allocation day & & & 0.780 \\
\hline G-CFF $(H z)$ & $41.1 \pm 2.4$ & $41.2 \pm 3.4$ & 0.073 \\
\hline R-CFF $(H z)$ & $40.8 \pm 2.6$ & $40.2 \pm 2.9$ & 0.666 \\
\hline CRT (milliseconds) & $544.4 \pm 86.6$ & $552.3 \pm 72.1$ & 0.661 \\
\hline HST error count & $79.4 \pm 26.3$ & $76.9 \pm 29.9$ & 0.279 \\
\hline Crossover day & & & 0.375 \\
\hline G-CFF (Hz) & $40.9 \pm 2.6$ & $40.6 \pm 2.7$ & 0.559 \\
\hline R-CFF (Hz) & $40.7 \pm 2.4$ & $40.4 \pm 2.3$ & 0.670 \\
\hline CRT (milliseconds) & $501.4 \pm 59.0$ & $489.2 \pm 86.0$ & \\
\hline HST error count & $82.8 \pm 20.9$ & $79.8 \pm 24.1$ & \\
\hline
\end{tabular}

Table 4: Subjective ratings of sleep and mood in decaffeinated and regular coffee group during post-lunch period.

\begin{tabular}{|c|c|c|c|c|c|}
\hline \multirow[b]{2}{*}{ Subjective mood parameters } & \multicolumn{2}{|c|}{ Decaffeinated coffee } & \multicolumn{2}{|c|}{ Regular coffee } & \multirow[b]{2}{*}{$P$ value } \\
\hline & $\begin{array}{l}\text { Pre-coffee } \\
(n=16)\end{array}$ & $\begin{array}{l}\text { Post-coffee } \\
(n=15)\end{array}$ & $\begin{array}{l}\text { Pre-coffee } \\
(n=16)\end{array}$ & $\begin{array}{l}\text { Post-coffee } \\
(n=16)\end{array}$ & \\
\hline Sleepiness- awake $(0-10 \mathrm{~cm})$ & $4.6 \pm 2.5$ & $6.1 \pm 2.3$ & $5.7 \pm 2.0$ & $7.1 \pm 2.3$ & 0.334 \\
\hline Anxious- casual (0-10 cm) & $7.8 \pm 1.8$ & $7.1 \pm 2.3$ & $8.2 \pm 1.9$ & $8.8 \pm 1.4$ & 0.07 \\
\hline Happy-sad (0-10 cm) & $4.1 \pm 1.7$ & $3.8 \pm 2.0$ & $3.6 \pm 1.8$ & $2.8 \pm 1.7$ & 0.199 \\
\hline Lethargic-energetic $(0-10 \mathrm{~cm})$ & $5.1 \pm 2.1$ & $5.6 \pm 2.1$ & $5.2 \pm 2.5$ & $6.8 \pm 2.0$ & 0.208 \\
\hline
\end{tabular}

Values represent mean $\pm \mathrm{SD} ; \mathrm{p}$ value listed for the post-coffee values comparison

\section{Subjective sleepiness and mood ratings during post- lunch period}

Mean scores of previous night sleep quality between decaffeinated and regular coffee groups were found to be $8.3 \pm 1.7$ and $7.8 \pm 1.8$ and the difference was not statistically significant $(\mathrm{p}=0.479)$. Evaluation of subjective rating of sleep has shown that twelve out of sixteen participants were found sleepy at pre-coffee time having visual analogue score of less than 5. Mean sleepiness-awake score was found to increase from pre-coffee time to post-coffee time in both the groups though the difference was not statistically significant $(\mathrm{p}=0.334)$. Anxious-casual score was marginally decreased in decaffeinated group and increased in regular coffee group from the pre-coffee scores. Like sleepiness-awake score, the happy-sad and lethargic-energetic scores showed a slight improvement in post-coffee values (Table 4).

\section{Adverse events}

None of the participants reported any adverse effects.

\section{DISCUSSION}

Post-lunch dip in both cognitive and physical performance in individuals during afternoon hours has been a commonly observed phenomena that has been more likely to occur in individuals who wake up very early in the morning. ${ }^{1,2,17}$ Such an effect has also been attributed to the high carbohydrate content of lunch. ${ }^{1}$ It is a usual practice to consume coffee or tea to counteract this lassitude. The present study was carried out to evaluate the effect of caffeine on psychomotor performance in post-lunch period in healthy volunteers having low to moderate caffeine intake. A randomised study was designed and the participants were given decaffeinated coffee or regular coffee in a cross over manner. As both quantity and nature of lunch have been reported to affect psychomotor performance, the participants were given uniform lunch on both allocation and crossover study days unlike previous studies. ${ }^{2,5}$

An evaluation of psychomotor function revealed that effect of lunch on psychomotor function on both the days revealed that the pre-lunch values for G-CFF, R-CFF, CRT and HST error count were comparable to the post-lunch i.e., pre-coffee values. In contrast to several other studies, a post-lunch dip in psychomotor performance was not observed in present study though a subjective feeling of sleepiness was present in most of the participants as also reported by others. ${ }^{2,5,17}$ This might be explained by the fact that laboratory-based performance activities might not universally show dip in post-lunch when compared to reallife settings. ${ }^{1}$

In present study both regular coffee (containing 90-100 mg caffeine) and decaffeinated coffee (containing 3-12 mg caffeine) were given with milk and sugar in accordance with regular practice in South Asian countries particularly in India. ${ }^{18}$ Intake of coffee in this form did not affect any of the psychomotor functions evaluated in this study to a significant extent when compared with pre-coffee values. Addition of milk to coffee have been shown to not to affect pharmacokinetics of coffee while addition of non-dairy creamer and sugar have been reported to decrease $C_{\max }$ and $\mathrm{T}_{\max }$ of coffee phenolic acids like caffeic acid and ferulic acid. $^{12}$ 
Caffeine given as black coffee or addition of caffeine capsule to decaffeinated coffee has earlier been reported to improve psychomotor performance..$^{2,11,19,20}$ It is quite possible that higher content of caffeine, other bioactive constituents of coffee or its aroma could have produced beneficial effect on psychomotor functions in previous studies. ${ }^{2,9,19}$ Frequent consumption of coffee during the day has also been shown to improve CFF and CRT at different time points of the day including afternoon when compared with plain water as placebo. ${ }^{10}$ Similarly, a single high dose of caffeine has also been reported to increase HST error count when compared with low dose..$^{21,22}$ In present study, the dose of caffeine administered in regular coffee group was lower in comparison to other studies. ${ }^{2,19}$

A slight improvement in sleep and mood rating after single cup of both decaffeinated and regular coffee as observed in present study is noteworthy. Both the coffee groups were more awake, felt more energetic and happier in comparison to findings of other studies. ${ }^{2,9} \mathrm{~A}$ slight positive effect of caffeine was evident on anxious-casual rating in regular coffee group which was not in line with its stimulant effect on autonomic nervous system. ${ }^{23}$ Caffeine has earlier been reported to improve mood and psychomotor performance in comparison to placebo. Besides, other bioactive compounds of coffee and the conditioning effect associated with coffee may also contribute to its beneficial effect on these parameters. ${ }^{8,24,25}$

The adjustment for baseline pre-coffee values and precoffee sleep and mood ratings on the primary outcome of psychomotor parameters did not yield any significant trend in the present study (data not shown). The effect of coffee on cardiovascular parameters in both the groups was not significant at the doses consumed in present study though higher doses of coffee have been shown to consistently increase both SBP and DBP in normotensive individuals. ${ }^{26}$

\section{Limitations}

The exact amount of caffeine in the final regular and decaffeinated coffee preparation were not estimated and the effect of milk and sugar on the bioavailability of caffeine could not be ascertained as the plasma caffeine levels were not measured in the participants. Participant's adherence to study instructions like avoidance of caffeine products were confirmed verbally; assessments of baseline caffeine levels were not done.

\section{CONCLUSION}

The present study shows that a single cup of regular or decaffeinated coffee in mild to moderate caffeine users when consumed after lunch may slightly improve subjective rating of mood and sleepiness but does not objectively affect psychomotor functions. Further investigation into examining the effects of bioactive components in coffee other than caffeine on the psychomotor functions is warranted.
Funding: No funding sources

Conflict of interest: None declared

Ethical approval: The study was approved by the Institutional Ethics Committee

\section{REFERENCES}

1. Monk TH. The post-lunch dip in performance. Clin. Sports Med. 2005;24(2):e15-23.

2. Smith AP, Rusted JM, Eaton-Williams P. Effects of caffeine given before and after lunch on sustained attention. Neuropsychobiology. 1990;23(3):160-3.

3. Quinlan PT, Lane J, Moore KL. The acute physiological and mood effects of tea and coffee: The role of caffeine level. Pharmacol Biochem Behav. 2000;66(1):19-28.

4. Smith AP, Sturgess W, Gallagher J. Effects of a low dose of caffeine given in different drinks on mood and performance. Hum Psychopharmacol Clin Exp. 1999;14(7):473-82.

5. Smith AP, Rusted JM, Savory M. The effects of caffeine, impulsivity and time of day on performance, mood and cardiovascular function. J Psychopharmacol Oxf Engl. 1991;5:120-8.

6. Zhou A, Taylor AE, Karhunen V. Habitual coffee consumption and cognitive function: a Mendelian randomization meta-analysis in up to 415,530 participants. Sci Rep. 2018;8:7526.

7. Lieberman HR, Tharion WJ, Shukitt-Hale B. Effects of caffeine, sleep loss, and stress on cognitive performance and mood during U.S. Navy SEAL training. Sea-Air-Land. Psychopharmacology (Berl.). 2001;164(3):250-61.

8. Camfield DA, Silber BY, Scholey AB. A randomised placebo-controlled trial to differentiate the acute cognitive and mood effects of chlorogenic acid from decaffeinated coffee. PloS One. 2013;8(12):e82897.

9. Dawkins L, Shahzad FZ, Ahmed SS. Expectation of having consumed caffeine can improve performance and mood. Appetite. 2011;57(3):597-600.

10. Hindmarch I, Rigney U, Stanley N. A naturalistic investigation of the effects of day-long consumption of tea, coffee and water on alertness, sleep onset and sleep quality. Psychopharmacology (Berl.). 2000;149(3):203-16.

11. Haskell-Ramsay CF, Jackson PA, Forster JS. The acute effects of caffeinated black coffee on cognition and mood in healthy young and older adults. Nutrients. 2018;10(10):1386.

12. Renouf M, Marmet C, Guy P. Nondairy creamer, but not milk, delays the appearance of coffee phenolic acid equivalents in human plasma. $J$ Nutr. 2010;140(2):259-63.

13. NHIS - Adult Tobacco Use - Glossary (2019). Available at: https://www.cdc.gov/nchs/nhis/tobacco/tobacco_glos sary.htm. Accessed on 23 March 2021.

14. Meredith SE, Julian LM, Hughes JR. Caffeine Use Disorder: A Comprehensive Review and Research Agenda. J Caffeine Res. 2013;3(3):114-30. 
15. Bordeaux B, Lieberman HR. Caffeine content in foods and beverages. Available at: https://www.uptodate.com/contents/image?imageKey $=\mathrm{OBGYN} \% 2 \mathrm{~F} 79304 \&$ topicKey $=\mathrm{PC} \% 2 \mathrm{~F} 5369 \&$ searc $\mathrm{h}=$ coffee\&rank=1 150\&source=see_link. Accessed on 27 March 2021.

16. Luczak A, Sobolewski A. The relationship between critical flicker fusion frequency (CFFF) and temperamental characteristics. Int J Occup Saf Ergon. 2000;6(4):493-505.

17. Smith AP, Miles C. The effects of lunch on cognitive vigilance tasks. Ergonomics. 1986;29(10):1251-61.

18. Executive Summary of Study on Domestic Consumption of Tea in India. Available at: http://www.teaboard.gov.in/pdf/Executive_Summary _Tea_Consumption_200 62018_pdf5940.pdf. Accessed on 27 March 2021.

19. Smith AP, Kendrick AM, Maben AL. Effects of breakfast and caffeine on performance and mood in the late morning and after lunch. Neuropsychobiology. 1992;26(4):198-204.

20. Irwin C, McCarty D, KhalesiS, Desbrow B. Caffeine Content and Perceived Sensory Characteristics of Pod Coffee: Effects on Mood and Cognitive Performance. Curr Res Nutr Food Sci J. 2018;6(2):329-45.

21. Bovim G, Naess P, Helle J. Caffeine influence on the motor steadiness battery in neuropsychological tests. J. Clin. Exp. Neuropsychol. 1995;17(3):472-6.
22. Miller LS, Lombardo TW, Fowler SC. Caffeine, but not time of day, increases whole-arm physiological tremor in non-smoking moderate users. Clin Exp Pharmacol Physiol. 1998;25(2):131-3.

23. McLellan TM, Caldwell JA, Lieberman HR. A review of caffeine's effects on cognitive, physical and occupational performance. Neurosci Biobehav Rev. 2016;71:294-312.

24. Shukitt-Hale B, Miller MG, Chu YF. Coffee, but not caffeine, has positive effects on cognition and psychomotor behavior in aging. Age (Dordr). 2013;35(6):2183-92.

25. Robelin M, Rogers PJ. Mood and psychomotor performance effects of the first, but not of subsequent, cup-of-coffee equivalent doses of caffeine consumed after overnight caffeine abstinence. Behav Pharmacol. 1998;9(7):611-8.

26. Nurminen ML, Niittynen L, Korpela R. Coffee, caffeine and blood pressure: a critical review. Eur J Clin Nutr. 1999;53(11):831-9.

Cite this article as: Ramachandran SS, Kumar VL, Dwivedi SN, Singh V, Gupta P. The post-lunch effect of regular and decaffeinated coffee on psychomotor performance: a randomized doubleblind cross-over study. Int J Basic Clin Pharmacol 2021;10:954-60. 